\title{
A Recent Case Reports on Unfamiliar Kikuchi-Fujimoto Disease
}

\section{Jasleen Kaur, Atif Khurshid Wani, Bijay Kumar Yadav* and Bhuban Subedi}

School of Biosciences and Bioengineering, Lovely Professional University, Phagwara, Punjab, India

*Corresponding author: Bijay Kumar Yadav, School of Biosciences and Bioengineering, Lovely Professional University, Phagwara, Punjab-144411, India, Tel: +7052053109; E-mail: jasleen12016@gmail.com

Received date: February 17, 2019; Accepted date: March 07, 2019; Published date: March 14, 2019

Citation: Kaur J, Wani AK, Yadav BK, Subedi B (2019) A Recent Case Reports on Unfamiliar Kikuchi-Fujimoto Disease. Med Clin Rev Vol. 5 No. 1 : 2.

Copyright: (02019 Kaur J, et al. This is an open-access article distributed under the terms of the Creative Commons Attribution License, which permits unrestricted use, distribution, and reproduction in any medium, provided the original author and source are credited.

\section{Abstract}

Kikuchi-Fujimoto Disease (KFD) is also known as Histiocytic Necrotizing Lymphadenitis. It is very rare, noncancerous and self-limiting Auto-Immune disease but mostly suspected as cancer, tuberculosis and systemic lupus erythematosus. It has symptoms such as cervical lymphadenopathy (enlargement of lymph nodes), low fever, headache, fatigue, night sweats and muscles pain. It typically affects young females aged between 20-35 years and mostly affects Asian populations. The main identification of KFD is characterized by Fine Needle Aspiration Cytology by taking blood sample from the swollen lymph node. Diagnosis is also possible with Fine Needle Aspiration Biopsy. Treatment for this disease has not been established. Non-steroidal Anti-Inflammatory Drugs or steroids are in use to ease the tenderness of lymph node and fever.

Keywords: Kikuchi-Fujimoto disease; Cervical lymphadenopathy; Histiocytic necrotizing lymphadenitis; Systemic lupus erythematosus; Lympho-histiocytic

\section{Introduction}

Kikuchi-Fujimoto (KFD) is benign, self-limiting auto-immune disease which is characterized by lymphadentis and continuous fever. It is also named as Histiocytic Necrotizing Lymphadenitis. The origin and pathogenesis of this disease has not been described yet. For the first time it was described by Kikuchi and Fujimoto in the year 1972 in Japan. It is a rare lympho-histiocytic disorder that affects mainly females of Asian region between the age group of 20-35 years. It has a ratio of $1: 2$ of the male and female respectively. It has been accounted in different countries but $80 \%$ of the cases were reported in Japan. Previously, it was predicted as viral infections such as human herpes virus (HHV) and parvovirus B19 [1].

Reports of the patients suggested that the pathogenesis might be auto-immune disease. The typical symptoms of KFD are lymphadenopathy, fever, muscle pain, night sweats and weight loss. It is widespread among young female adults in Asia. It is a non-cancerous disorder but occasionally it leads to misdiagnosis such as tuberculosis, rheumatologic and hematologic malignancies. Morphologic examination of the swollen lymph nodes is right path of correct diagnosis in KFD, however, the recognition by morphology is a challenge to many pathologists [2].

\section{Three fundamental histological stages of disease}

Proliferative stage: Expresses various monocytes, plasmacytoid, lymphoid cells and histiocytes which contain Karyorrhetic Nuclear Fragments.

Necrotizing stage: Expresses an extent of coagulative necrosis.

Xanthomatous stage: Overstuffed with the lathered histiocytes [3].

\section{Symptoms}

Lymphdenitis (Painful lymph nodes with swelling); Lymph Node non-tender and rubbery; Mild shortness of breath; Night sweats, fatigue, nausea and vomiting; High Fever (Continuous); Muscles pain, headache and weight loss; Cough [4].

\section{Clinical features}

Clinical features are shown by the occurrence of cervical lymphadenopathy (affecting the lymph nodes) in about $90 \%$ cases, which is usually present in trapezius and carotid lymph nodes and observed in patients. Lymph nodes become swollen, sometimes large measuring from 2 to $6 \mathrm{~cm}$ in diameter and painful. Systemic sign includes pain in the muscles, nausea, fever etc. and has been found in approximately $30 \%$ to $50 \%$ of the cases. These often led to misdiagnosis as malignancy or cancer. A hepatosplenomegaly (enlargement of liver and spleen), pseudo-urticarial rash, arthralgia, loss of weight etc. might also be common in the patient [5]. 
The disease weakens the immune system and may usually last for few months to year or even more. Patients recovery may take a longer duration [6].

\section{Cause and pathogenesis}

It has been in doubt whether KFD is a viral or autoimmune disease. Some researchers suggested that it is caused due to virus but some suggested it as an auto-immune disease. KFD is an uncommon and very rare disease. Based on the clinical appearance infectious causes have been hypothesized particularly as viruses due to the clinical features. Some seditious agents have been diagnosed such as Virus of human Herpes, Virus of Epstein-Barr. However, they failed to confirm the causative link between these pathogenic agents and the KFD.

On the basis of clinical appearance and laboratory research features, it was thought that KFD may show $\mathrm{T}$ cell associated hyper-response to some antigen stimulus in genetically prone persons. But some of the studies have put forth that primary cells might be the CD8+ T lymphocytes, which provoke apoptosis, some indicate that degree of inflammatory mediators, such as interleukin 6 supports an immunemediated ethology [7].

\section{Viral infections}

Human Herpes Virus, Epstein Barr Virus, HIV and Parvovirus B19 are the most studied viruses in KFD patients. KFD

How it was excluded that viruses were not the cause of

Human herpes viruses: Approximately 15 samples of KFD and 20 controls were studied by the Researchers by using Nested Polymerase Chain Reaction. They did not find any variation between the viral DNA sequences of controls and patients. The amplification of the sequences of Human Herpes Viruses from lymph nodes in 6 patients out of 24 was done. But they did not find any viral sequences in the reactive lymphadenopathy of the patients.

Epstein barr virus: From the patients with KFD around 30 lymph node samples and 12 controls were tested for the existence of Epstein Barr Viruses by using Real Time PCR. Some cells with apoptotic aspect positive for Epstein Barr Virus encoded RNA were found in the necrotic regions of the KFD patients. It was suggested that the disease might be due to the hyper immune reaction in opposition to Epstein Barr Infection. Many other cases of KFD patients were studied but did not find any role of virus in the pathogenesis of KFD.

Overall, the consequences of the studies which were for the viral cause of Kikuchi have been not compatible. Different laboratory methods were used but in very few numbers of samples were found positive results and confirmatory studies with the control samples were frequently unavailable [8].

\section{Case Series}

\section{Case 1}

In 2016, a girl aged 20 years was presented in Holy Family Hospital, New Delhi, who was suffering with fever, weight loss and 4 enlarged lymph nodes for last three weeks. Until that time, she had been fit and healthy and was on no medication. On examination, significant findings were on lymphadenopathy, posterior auricular and cervical. On examination $2 \mathrm{~cm}$ smooth and tender lymph node lumps were found near the left ear.

CBC (Complete Blood Count) Test was performed which was found negative ANA (Anti-Nuclear Antibody) Tests were also performed to evaluate the patient for the auto-immune disorder which are systemic (affect many organs and tissues in the body) but all were found to be negative. During this period, she was given the Augmentin Antibiotic until it was diagnosed.

Doctors of Holy Family Hospital suspected the patient for tuberculosis and patient was made to undergo for Mantoux Tuberculin Skin Test. But the result was found to be negative. In chest X-ray, infection was also found. This case was becoming critical and serious.

At last, doctors suggested for FNAC test. On examination reactive lymphadenopathy was found. Then, this case was misdiagnosed as cancer but before the biopsy doctors had meeting with the senior doctors in which it was diagnosed as Kikuchi Fujimoto.

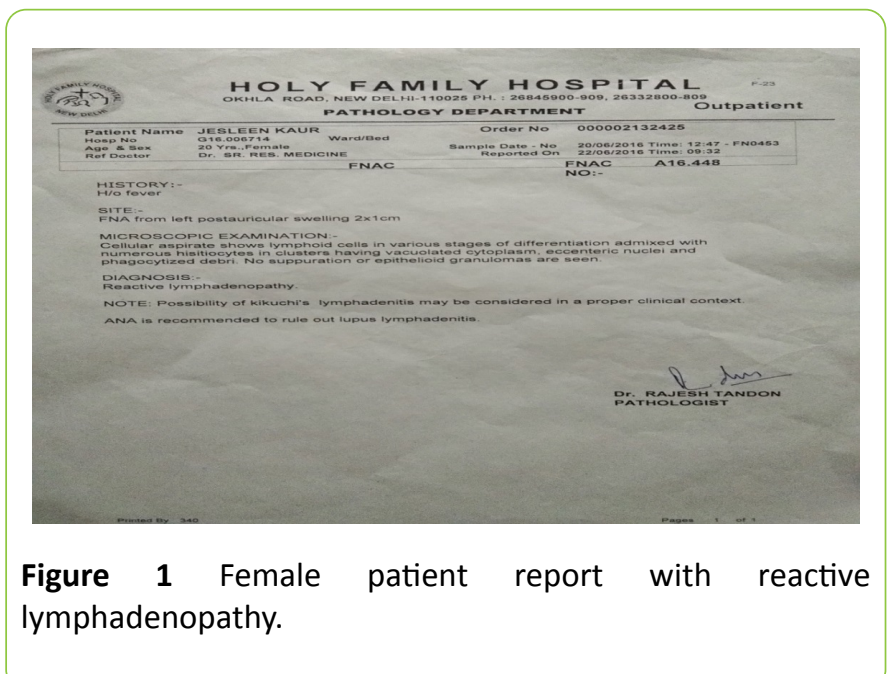

Discussion: In India, it was one of the rare cases of Kikuchi Fujimoto. The patient became very weak and infection spread in her neck. After diagnosis, she had been under medication and had a regular check-up for lymph nodes for about 1 year (Figure 1).

Medication available: Dose of Wysolone tablet, a type of corticosteroids was given to the patient. Dose was increased from $5 \mathrm{mg}$ to $15 \mathrm{mg}$ for induction therapy. She had initially taken for 6 months. This steroid had side effects like such as 
anxiety, depression and sleeplessness and others like weight gain, muscle and bone aches and pains etc.

Pantocid 40 was given to treat gastric problems that emerged with treated drug; the D3 solution to prevent the deficiency of vitamin $D$.

Finally, she recovered from the disease with all these medications 1 year but the side effects remained for more 1 year.

\section{Case 2}

In 2012, a female under the age of 32 was examined to have the swollen and enlarged lymph nodes on both sides of her neck with no pain and no weight loss. An excision biopsy was performed and submitted for histopathological assessment. After observing under microscopy, an extensive area of necrosis bounded by the broad zones of histiocytes and activated lymphoid cells were found. In some other areas of the nodes, expansions of Para cortical regions were seen. The small reactive lymphoid cells in the swollen lymph nodes were also present. Histopathology suggested a necrotizing lymphadenitis which was suspected as systemic lupus erythematosus (SLE). Other tests were performed such as ANA, but the results were negative. Patient was treated with symptomatic treatment for weeks with Wysolone, Pentocid 40 and Augmentin [9].

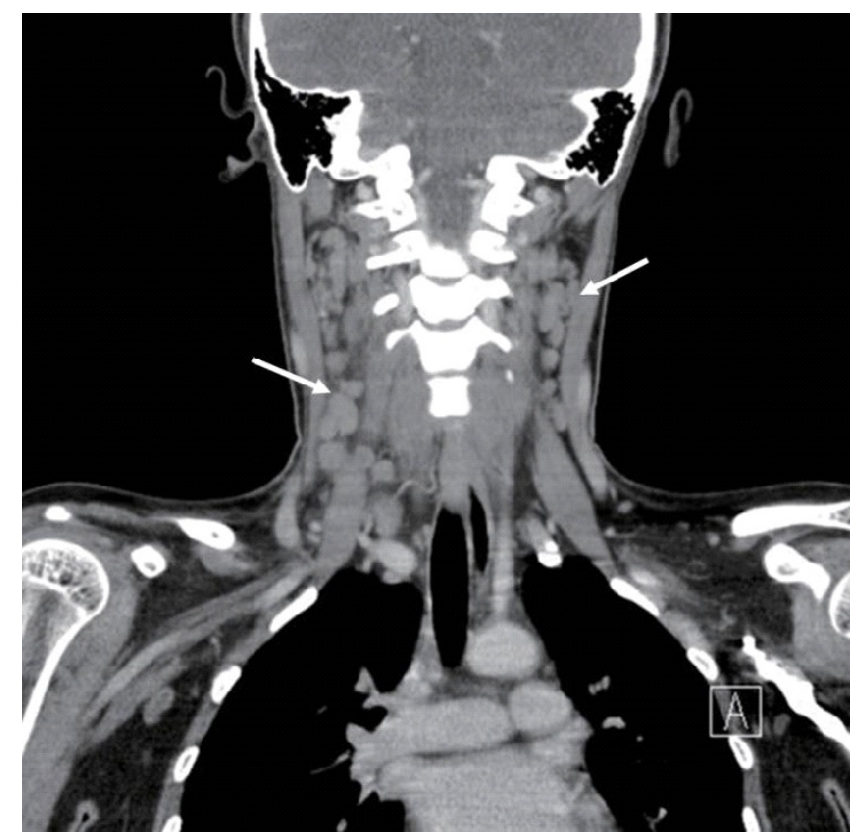

Figure 2 Computed tomography of the neck shows multiple enlarged lymph nodes on both sides of the neck.

Discussion: If we look at the countries where Kikuchi female patients were reported, three females were affected with KFD in United States (2008). In Japan (2017) one of the females was reported with this disease. In Italy (2016), 66 years old female was suffered with two months history of fever which was diagnosed as Kikuchi (Figure 2).

\section{Case 3}

A man aged 20 years was admitted in hospital with fever, night sweats, muscle pain, weight loss and lymphadenopathy for about three weeks. Complete blood count test demonstrated a WBCs count $2.1 \times 10^{9} / \mathrm{L}$, neutrophils $48 \%$, lymphocytes $39 \%$, monocytes $8 \%$, eosinophils $8 \%$ and platelet count $87 \times 10^{9} / \mathrm{L}$. Tomography scan demonstrated unclear hyper-metabolic left lymph nodes with a diameter of $1.8 \mathrm{~cm}$. Lymph node biopsy report showed very reactive lymphoid with prolonged paracortical karyorrhexis, paracortex and necrosis. Lymph node swelling decreased and become normal within 6 months after regular check-up and treatment. The treatment was supportive and resolution of lymph node occurred within 6 months. This disease is non-cancerous disorder with unknown cause, described by common symptoms of leucopenia [10].

\section{Case 4}

A 36-year-old man from UK complained about two weeks of continuous fever, painful knee joint, night sweats and headache. He had no past medical history. His body temperature was $38.2^{\circ} \mathrm{C}$ and heart beat rate 105 beats/min. He had cervical lymphadenopathy and swelling in his lymph nodes. Lymph nodes were hard and non -tender. Pharynx examination was also normal.

Renal and liver tests were normal. Malarial parasite test was negative, there was no malarial parasite seen on blood film.

He was treated with antibiotics because of the unknown cause. Two salivary samples were taken which were found negative for the immunoglobulin $\mathrm{M}$. Urine cultures was sterile. Fever persisted for another two weeks. After that antibiotics were not given because there was no relief or improvement. NSAIDs were then prescribed. His cervical lymphadenopathy was still persistent. Through ultrasound it came to the knowledge that he had distended lymph in the neck.

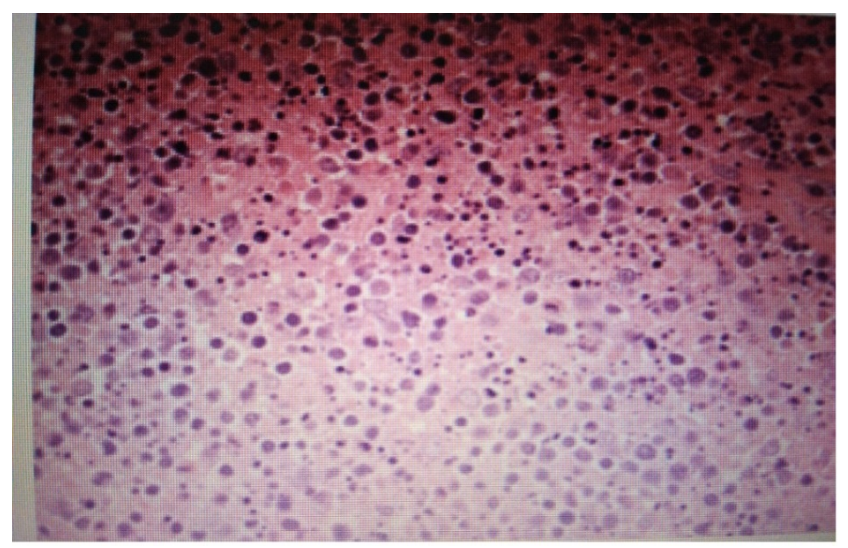

Figure 3 Showing individual cell death and nuclear debris in KFD patient.

An excisional biopsy of cervical lymph node discovered reactive hyperplasia. After diagnosis, medication was started 
and the patient felt better. Two months later he recovered completely (Figure 3) [11].

\section{Diagnosis}

Lymph node biopsy: FNAB (Fine-Needle Aspiration Biopsy) is a vital tool for the diagnosis of some infectious lymph-node disorders. This biopsy is the ultimate diagnostic tool used in patients those who have swollen lymph nodes. Approximately 44 cases have been analysed and reported with KFD using FNAB technique [12].

In this, whole swollen lymph node is detached and assessed under the microscope. Lymph node swells when they are infected. Lymph nodes are the small glands which make WBCs (lymphocytes) to fight against the infections [13].

Fine needle aspiration cytology: FNAC (Fine Needle Aspiration Cytology) of the swollen lymph node is easy to diagnose the KFD and to make the degree of malignancy clear. In this an unfilled needle is put into the lump present in the neck to collect a sample of cells which are stained, and these cells are examined under a microscope. It is a most powerful technique to diagnose Kikuchi and by this technique there are less chances of misdiagnosis [14].

Treatment: Treatment for this disease has not been fully established, and recommendations depend upon the reports and expert opinion only. Due to the rare and self-limiting disease it is very significant to observe the symptoms which can be quite serious. High dose glucocorticoids with intravenous immunoglobulin have been shown some good results in the severe symptoms.

NSAIDS (Non-steroidal Anti-Inflammatory Drugs) or steroids can also be used to ease the tenderness of the lymph node and fever.

Corticosteroids for example prednisone, has been suggested in severe cases or widespread Kikuchi disease. In some critical and complicated conditions, hydroxychloroquine is also being prescribed [15-19]

\section{Discussion and Conclusion}

Kikuchi Disease is an enormously uncommon and is usually misdiagnosed as cancer or tuberculosis; which involves the cervical lymph nodes. Kikuchi's disorder mostly spread in Asian and Japanese population. Few cases have been reported in India in 2016 and 2018.

This disease is not much dangerous and sometimes its symptoms resembles with other disease which leads to misdiagnosis such as tuberculosis, lymphoma. Early recognition of the disease is vital to avoid the misdiagnosis.

To avoid misdiagnosis, cognizance of this disease in the whole world is very necessary especially for the clinician as well as for the pathologists. KFD in some cases have been diagnosed with the lymph node biopsy technique have shown good outcome.
Earlier, FNAC was also used for its diagnosis. Now-a-days, there is no proper treatment found for it except the steroids, which would have various side effects.

For about 40 years before, even though KFD was little familiar among people but the cause of this disease is still unknown. It has been proposed that it might be autoimmune or infectious disease but have been not definitely confirmed. Clinical presentation with symptoms such as systemic and fever lead to its misdiagnosis. Till reliable prognostic makers are revealed, patients with KFD need to undergo regular check-ups due to their increased risk of Systemic Lupus Erythematosus. Even now this disease is very rare and no proper treatment is found. Its mild effect involves weakening of the over immune system.

\section{References}

1. Lelii M, Senatore L, Amodeo I, Pinzani R, Torretta S, et al. (2018) Kikuchi-Fujimoto disease in children: Two case reports and a review of the literature. Ital J Pediatr 44: 83

2. Lo KB, Papazoglou A, Chua L, Candelario N (2018) Case report: Kikuchi: The great mimicker. F1000Res 7: 520.

3. Abhinav B, Vimala R, Balaji P, Ramanakumar RV (2018) Histiocytic necrotizing lymphadenitis- kikuchi disease: An overview. PARIPEX- Ind J Res 7: 29-30.

4. Kulak J, Smith LP, Acar BC, Weed DT (2009) Kikuchi Fujimoto disease. Ear Nose Throat J 88: 1108-1109.

5. Farikha F, Marzouk S, Frigui M (2008) Maladie de kikuchifujimoto et connectivite: A propos de trios observations. Rev Med Interne 29: 129-134.

6. Kuo TT (2008) kikuchi's disease (histiocytic necrotizing lymphadenitis): A clinicopathologic study of 79 cases with an analysis of histologic subtypes. Am J Surg Pathol 19: 798-809.

7. Găman $M$, Vlădăreanu $A M$, Dobrea $C$, Onisâi $M$, Marinescu $C$, et al. (2018) A challenging case of Kikuchi-Fujimoto disease associated with systemic lupus erythematosus and review of the literature. Case Report Hematol pp: 1-5.

8. Deaver D, Horna P, Cualing H, Sokol L (2014) Pathogenesis, diagnosis, and management of Kikuchi-Fujimoto disease. Cancer Control 21: 313-321.

9. Nagarkar R, Adhav A (2012) Kikuchi Fujimoto disease: a rare benign disease. SAS J Sur 3: 138-140.

10. Xavier B, Guilabert A (2006) Kikuchi-Fujimoto disease. Orphan J Rare Dis 1: 18.

11. Wilson MR, Milne G, Vryonis E (2014) Kikuchi-Fujimoto disease: A rare cause of fever in the returning traveller. Case Report Med.

12. Deaver D, Naghashpour M, Sokol L (2014) Kikuchi-Fujimoto disease in the United States: three case reports and review of the literature. Mediterr J Hematol Infect Dis 6: e2014001.

13. Lin HC, Su CY, Huang CC, Hwang CF, Chien CY (2003) Kikuchi's disease: A review and analysis of 61 cases. Otoralyngol Head Neck Surg 128: 650-653.

14. Singh G, Iyer VK (2008) Fine needle aspiration cytology of Kikuchi's Disease Lippincott Williams and Wilkins. J Cytol 25: 36-37. 
15. Horna P, Deaver D, Cualing H, Sokol L (2014) Pathogenesis, diagnosis, and management of Kikuchi-Fujimoto disease. Cancer Control 21: 313-321.

16. Zaghloul A, lorgoveanu C, Polio A, Desai A (2018) KikuchiFujimoto disease in a young African American male. Cureus 10: e2508.

17. Dalugama C, Gawarammana IB (2017) Fever with lymphadenopathy-Kikuchi Fujimoto disease, a great masquerader: A case report. J Med Case Rep 11: 349.
18. Ohta EE, Kaizaki NY (2013) Axillary, intramammary lymphadenopathy caused by kikuchifujimoto disease mimicking malignant lymphoma. Mediterr J Hematol Infect Dis 20: 97-101.

19. Cunha BA, Mickail N, Durie N, Pherez FM, Strollo S (2009) Fever of Unknown Origin (FUO) caused by Kikuchi's disease mimicking lymphoma. Heart Lung 38: 450-456. 